\title{
Tracking and Minimization of Adverse Events in the Patient Care Process while in a Hospital Emergency Service Area ${ }^{\dagger}$
}

\author{
María Martínez Pérez *, Carlos Dafonte, Ana Belén Pérez Boado and Ángel Gómez \\ CITIC-Department of Computer Science, University of A Coruña, Campus de Elviña s/n, \\ 15071 A Coruña, Spain; carlos.dafonte@udc.es (C.D.); ana.perez@udc.es (A.B.P.B.); \\ angel.gomez@udc.es (Á.G.) \\ * Correspondence: maria.martinez@udc.es; Tel.: +34-981-167-000 (ext. 1264) \\ + Presented at the 13th International Conference on Ubiquitous Computing and Ambient Intelligence \\ UCAmI 2019, Toledo, Spain, 2-5 December 2019.
}

Published: 20 November 2019

\begin{abstract}
The aim of this work was to integrate and computerize into a single program the most important tasks in the process of prescription, dispensation, and administration of medication to patients during their stay in a hospital center. This will increase quality, efficiency, and safety in the care process. An interface was also developed that allows for real-time determination of the whereabouts of a patient within the emergency services unit and the implementation of solutions provided by identification technologies, such as RFID (Radio Frequency Identification), to calculate the location of a patient with more precision and at a reduced cost.
\end{abstract}

Keywords: traceability; emergency services; RFID

\section{Introduction}

It is nowadays essential to be able to track processes in a professional environment in order to improve the quality, efficiency, and safety of the tasks undertaken. Tracking is defined as the recording, from origin to destination, of any task undertaken which includes the key data and the personnel involved. It is important to highlight the advantages of conducting a subsequent and detailed analysis of the registered data in order to detect bottlenecks and undertake, where necessary, measures for improvement [1].

Tracking is fundamental in a health environment and is a primary concern of health professionals [2], in that it can reduce the occurrence of adverse events. An adverse event is understood as any complication that arises during a patient's hospital stay and which is not directly related to the base illness or reason for admission [3,4]. Implementing a tracking system in an accident and emergency unit is especially interesting given its specific characteristics and the challenges it presents for the health professionals involved. An accident and emergency unit is characterized by its high rotation of patients, changes of shift among health professionals, and the specific administrative procedures for patients [5].

The key points in obtaining traceability in the healthcare environment are focused on the unambiguous identification of the patient, medication, and protocols related to the care plan of the patient, so that the patient can be localized at any given time. A primary reason for this work is, therefore, to computerize the previously described tasks in addition to minimizing the need to use paper or the telephone.

In this way, the computerization of daily clinical practice allows health professionals access to the same updated data and provides a unique registry that reduces the possibility of human error, both in the understanding of the available data and its transcription at the time of updating. 
Prior to this development, a tool that computerizes the care process of the patient while in the emergency services unit did not exist, as most of the tasks were recorded on paper. This work, therefore, aimed to improve on those tasks undertaken in daily clinical practice. The detailed objectives are described below.

\section{Objectives}

The aim of this work was to develop a web application that allows various health professionals involved in the prescription, dispensation, and administration of medication to patients within an accident and emergency unit to carry out those tasks involved in the care process of the patient in an automated way that is rapid, efficient, and safe.

More specifically, the work that was carried out involved the following tasks:

- The indication of any pending changes in the location of patients to be carried out by hospital auxiliaries;

- The identification of the position of patients in real time;

- The prescription of treatment to patients on the part of the doctor;

- The administration of treatment to patients by nursing staff;

- The measurement of vital constants on the part of nursing staff;

- The control of techniques and complementary tests carried out by nursing staff.

Different health professionals (doctors, nurses, and auxiliary staff) have distinct roles within the application, which allows them to perform tasks for which they are authorized and provides them access to the necessary data.

Owing to our experience in integrating RFID technology to obtain patient and medication traceability [6-9], we designed a visual interface of the location and medication of a patient while in hospital. This interface had to have the appropriate characteristics in its design to easily integrate with a location motor, for example, a location motor which calculates the whereabouts of a patient and which is controlled by identification technologies such as RFID.

\section{Development}

This section focuses on the development stage of this project.

This work adapted the Scrum approach, which belongs to a set of development software methodologies called "Agile". One of its main benefits is that it provides a framework for work based on an iterative and incremental process, which is suitable for flexible projects that require adaptable and rapid execution. In addition, it generates, from the application, executables for each iteration, which allows continual revision of the developed software, as evidenced by the health personnel who collaborated on this project from the emergency services unit at the University Hospital of Santiago de Compostela. The main stages of this methodology were analysis, design, implementation, and testing.

The first stage entailed carrying out an analysis of how an emergency unit operates and determining the key points in the prescription, dispensation, and administration of medication in order to properly track them. To achieve this, various meetings were held with the different personnel involved at each stage to glean the detailed and necessary information involved in the particular processes. This involved the study of the following points: consultation of the documents used in the care process of the patient (see Figure 1), analysis of the interchange of information relating to the patient by the health professionals involved, and identification of the route of the patient within the emergency services unit (waiting area, nursing, critical service area, benches, examination boxes, treatment areas, and observation rooms). 


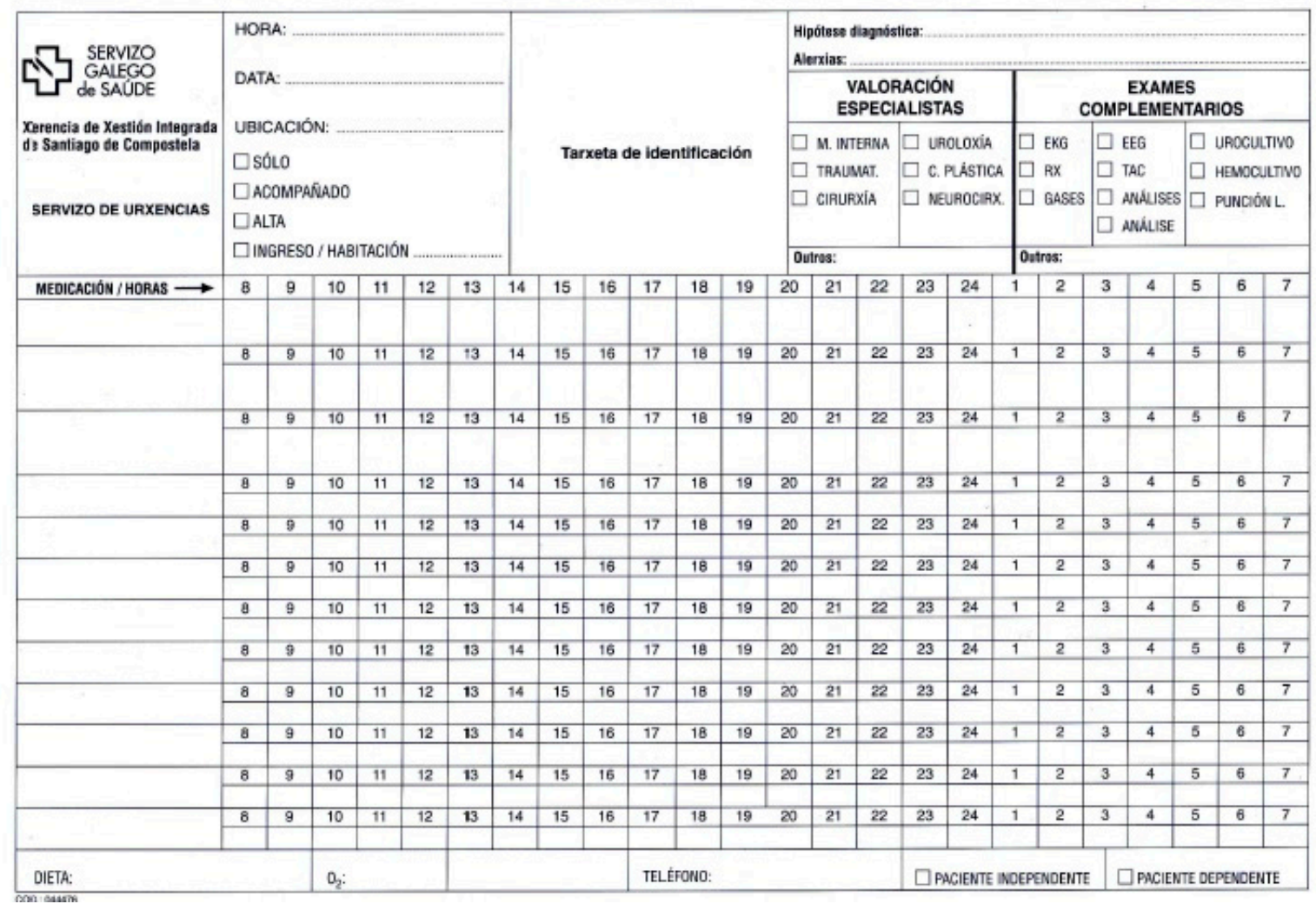

Figure 1. Medication administration record and complementary medical examinations.

This all contributed to developing the professional knowledge pool relating to the protocol for those tasks previously described, thus minimizing the learning time required on the part of those health professionals involved and improving the quality of care delivered to the patient while in the emergency services unit.

The second stage was the design phase, where a study of the appropriate technologies for the development of the tool was essential, as were the multiple versions of the interface before deciding on the definitive version. This stage involved all of the health personnel referred to in this work. The Scrum methodology was employed in the implementation, which enabled more fluid communication with clients and kept them informed of the progress and any changes in the project. The model-viewcontroller (MVC) architecture pattern was followed, which allowed for smooth adaptation when including additional functionalities or integrating it into other hospital services.

The application was developed with JavaEE, JDBC, CSS, JavaScript, and HTML technology, among others, with MySQL selected as the database manager. Hibernate was used to develop the model layer and the connection to the database. This allowed the developer to detail the database and its relations. In addition, a main feature is that it provides support for the model based on objects, which allows for a transparent mechanism for persistence. Spring provided a framework with a modular structure and great flexibility for the implementation of different types of architecture. Apache Tapestry was used as the framework to create open code web applications developed with Java language, based on components. This employed a modular approximation in web development and related the web component and its correspondent in Java (see Figures 2 and 3). 


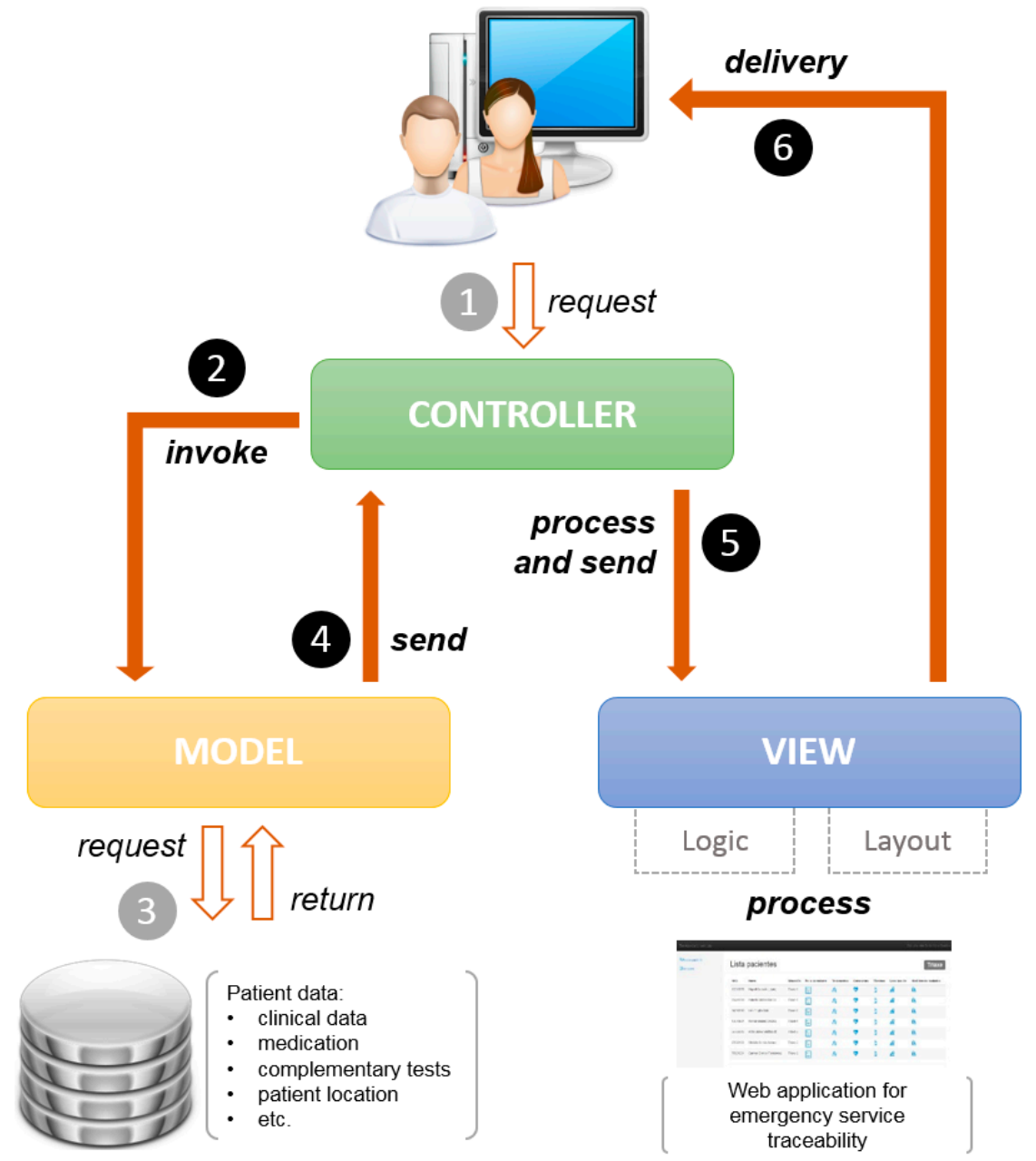

Figure 2. Model-view-controller pattern in the developed application.

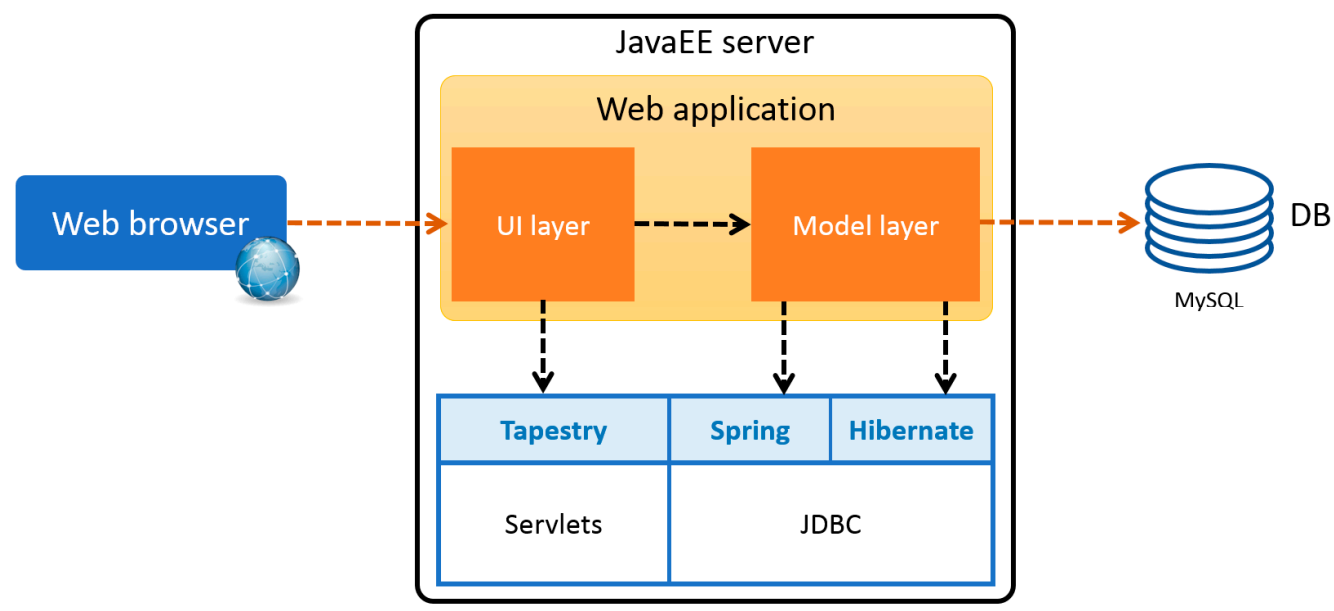

Figure 3. System architecture and technologies used in the system.

The application was implemented so that users can only carry out functionalities in line with their professional profile. Some of the more prominent and common functionalities for authorized users (doctors, nursing staff, and orderlies) are as follows: patient location view, traceability view of a patient, listing of patients in a specific location, and search location of a patient.

A common module exists for tasks involving both doctors and nursing staff. These activities include patient registration update, patient registration consultation, vital signs consultation, 
administered and pending medication consultation for a patient, and authorization for the transfer of a patient to another area.

The tool also allows doctors to carry out the following duties in line with their profile: modification of medication prescribed to a patient, indication of techniques/tests to be performed on a patient, and discharge or relocation of the patient to another service within the hospital complex.

Nursing staff can also carry out the following tasks in line with their profile: record/update and add patient vital signs information, register complementary tests and techniques carried out on the patient, and record medication administered to the patient.

Figures 4 and 5 respectively illustrate a doctor's list of patients, their location, and an example of the tasks that have been previously described.

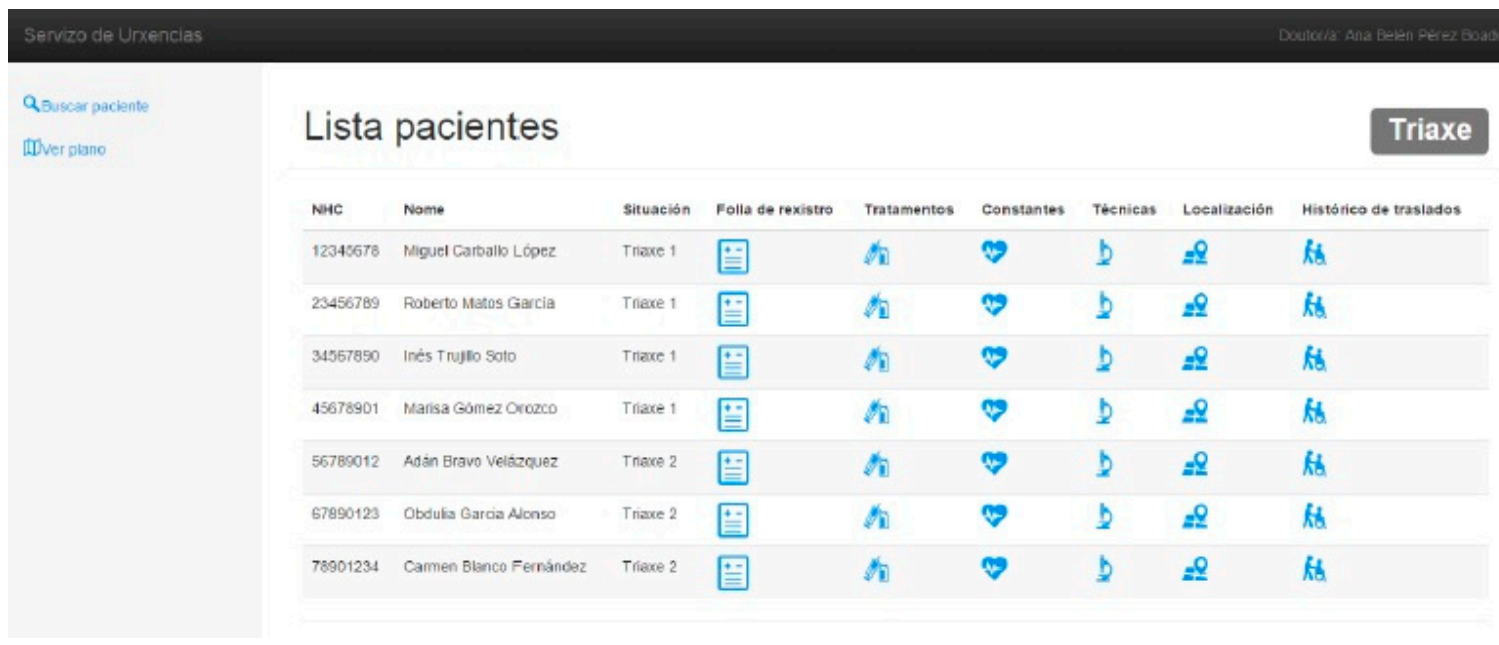

Figure 4. Patient list assigned to a doctor in different locations within the emergency services area.

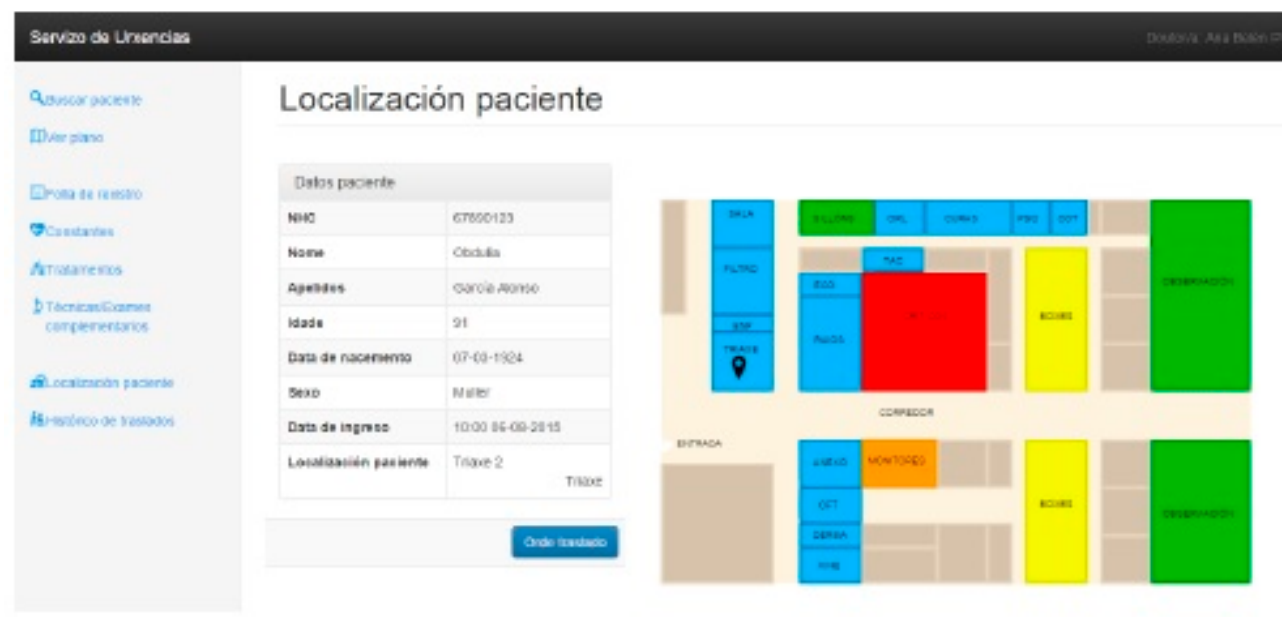

Figure 5. Emergency services area map for data acquisition and patient location.

Following its development, the application was considered as complete, given that it complied with the functional requirements previously described. The application was tested with unit and system tests and also functionally by health professionals involved in the project. It was tested directly by at least one specialist representing each professional profile involved in this project, whose contribution was essential for the successful outcome of the work. 


\section{Discussion and Conclusions}

This work complied with the objectives previously described and met the expectations of the health professionals involved. The success of the tool, with respect to its efficiency and usability, was largely determined by the active participation of those health professionals from the start to finish of the project. Their participation led to refinement at each iteration in the life cycle of the product, resulting in the final version.

The implemented interface which views, in real time, the location of the patient in each of the emergency service areas has an optimum design, into which the data provided by a location motor, which automatically calculates the position of a patient with greater precision by using technologies such as RFID, can be integrated. This avoids costs associated with proprietary license software supplied by hardware manufacturers for the adopted solution [6-9].

This application improves quality in both the care and the prescription, dispensation, and administration of medication processes and significantly aids the traceability of a patient within a hospital, resulting in an increase in both efficiency and safety.

This has been made possible for two reasons: the automatization of the activities involved in the process and the decrease in the possibility of human error. This significantly reduces the occurrence of adverse events during a patient's hospital stay.

The knowledge provided by the specialists during the analysis stage allowed us to replicate, to a large extent, the previous protocol (the computerization of document models, for example) and develop this tool in such a way that the learning time required to use the tool is reduced considerably. This has significantly contributed to the degree of satisfaction that the health personnel have for the application.

Author Contributions: M.M.P., C.D., A.B.P.B., and Á.G. conceived, designed, and performed the experiments and developed all the tools. The paper was written by all of them.

Funding: This research was funded by the Xunta de Galicia (grant number Potencial Crecemento ED431B 2018/42), the European Union (European Social Fund-ESF) and the Spanish Ministry of Science, Innovation and Universities and the Ministry of Economy, Industry and Competitiveness (grant numbers RTI2018-095076B-C22 and ESP2016-80079-C2-2-R).

Acknowledgments: This work was supported by the Xunta de Galicia (Potencial Crecemento ED431B 2018/42) and the European Union (European Social Fund-ESF); we also used IT infrastructure that was acquired through the RTI2018-095076-B-C22 and ESP2016-80079-C2-2-R projects, financed by the Spanish Ministry of Science, Innovation, and Universities and the Ministry of Economy, Industry, and Competitiveness.

Conflicts of Interest: The authors declare no conflict of interest.

\section{References}

1. ISO 9000:2015. Sistemas de Gestión de la Calidad. Available online: https://www.iso.org/obp/ui/es/\#iso:std:iso:9000:ed-4:v1:es (accessed on 25 June 2019).

2. Osborn, S.; Williams, S. Seven steps to patient safety. In An Overview Guide for NHS Staff, 2nd ed.; The National Patient Safety Agency: London, UK, 2004.

3. Kohn, L.T.; Corrigan, J.M.; Donaldson, M.S. To Err Is Human: Building a Safer Health System; Institute of Medicine National Academy Press: Washington, DC, USA, 1999.

4. Leape, L.L.; Brennan, T.A.; Laird, N.; Lawthers, A.G.; Localio, A.R. The nature of adverse events in hospitalized patients. Results of the Harvard Medical Practice Study II. N. Engl. J. Med. 1991, 324, 377-384.

5. Martínez Pérez, M.; Cabrero-Canosa, M.; Hermida, J.V.; García, L.C.; Gómez, D.L.; González, G.V.; Herranz, I.M. Application of RFID technology in patient tracking and medication traceability in emergency care. J. Med. Syst. 2012, 36, 3983-3993.

6. Martínez Pérez, M.; Dafonte, C.; Gómez, Á. Traceability in Patient Healthcare through the Integration of RFID Technology in an ICU in a Hospital. Sensors 2018, 18, 1627.

7. Martínez Pérez, M.; Vázquez González, G.; Dafonte, C. Safety and Traceability in Patient Healthcare through the Integration of RFID Technology for Intravenous Mixtures in the Prescription-ValidationElaboration-Dispensation-Administration Circuit to Day Hospital Patients. Sensors 2016, 16, 1188. 
8. Martínez Pérez, M.; Vázquez González, G.; Dafonte, C. Evaluation of a Tracking System for Patients and Mixed Intravenous Medication Based on RFID Technology. Sensors 2016, 16, 2031.

9. Martínez Pérez, M.; Vázquez González, G.; Dafonte, C. The Development of an RFID Solution to Facilitate the Traceability of Patient and Pharmaceutical Data. Sensors 2017, 17, 2247. article distributed under the terms and conditions of the Creative Commons Attribution (CC BY) license (http://creativecommons.org/licenses/by/4.0/). 